\title{
Cronologías de ancho de anillos de queñoa (Polylepis tarapacana) para los últimos 500 años en el Altiplano de la región de Arica y Parinacota, Chile
}

\author{
Tree rings Chronologies of queñoa (Polylepis tarapacana) for the last 500 years in the Altiplano of \\ Arica and Parinacota Region, Chile
}

\author{
Jorge Moya ${ }^{a *}$, Antonio Lara ${ }^{b}$ \\ * Autor de correspondencia: ${ }^{\text {a } U n i v e r s i d a d ~ A u s t r a l ~ d e ~ C h i l e, ~ I n s t i t u t o ~ d e ~ M a n e j o ~ F o r e s t a l, ~ L a b o r a t o r i o ~ d e ~ G e o m a ́ t i c a, ~ c a s i l l a ~ 567, ~}$ \\ Valdivia, Chile, tel.: +56 63 293357, jorgemoyarossi@gmail.com

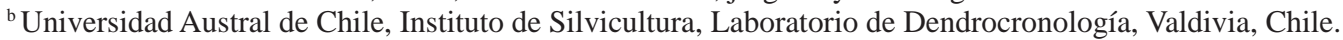

\begin{abstract}
SUMMARY
The need to cover the dendrochronological research gaps in the Sub Tropical regions of South America motivated the search for new geographical areas and new species to investigate climatic variability. Polylepis tarapacana grows above 4,200 m a.s.l. in South America $\left(16^{\circ}-23^{\circ} \mathrm{S}\right)$, permitting the expansion of dendrochronological studies in subtropical areas. Samples from living and dead trees were collected in January 2003, near Nasahuento village ( $17^{\circ} 32^{\prime} \mathrm{S}, 69^{\circ} 35^{\prime} \mathrm{W}$ ) at 4,313 m a.s.l. and Guallatire volcano (18 $48^{\prime}$ $\mathrm{S}, 6^{\circ} 08^{\prime} \mathrm{W}$ ) at $4,750 \mathrm{~m}$ a.s.l. These samples rendered the first two tree-ring chronologies of P. tarapacana in Chile. Nasahuento and Guallatire chronologies date back 242 and 536 years, respectively. Both were compared with instrumental records of precipitation and temperature. Statistics obtained from these analyses showed that both sites are adequate to be used for dendroclimatic purposes. Correlation functions showed that growth is mainly regulated by precipitation, correlating positively with the previous summer and negatively with the current one. January shows the most significant coefficients for both years. On the other hand, temperature is negatively correlated with the previous summer and positively correlated with the current season, evidencing a relationship between tree growth and water availability. Tree-ring chronologies developed in this study provide high-resolution climate sensitive records that are valuable for future climate reconstructions.
\end{abstract}

Key words: Polylepis tarapacana, dendrochronology, climatic variability.

\section{RESUMEN}

La necesidad de llenar vacíos dendrocronológicos en regiones subtropicales de Sudamérica motivó la búsqueda de nuevas áreas y especies capaces de registrar en sus estructuras leñosas la variabilidad climática. La presencia de comunidades arbóreas/arbustivas de Polylepis tarapacana, creciendo sobre 4.200 m s.n.m. en la región alto andina sudamericana $\left(16^{\circ}-23^{\circ} \mathrm{S}\right)$, permitió incorporar nuevas áreas para estudios dendrocronológicos. En enero de 2003 se colectaron muestras dendrocronológicas de árboles vivos y muertos, en las cercanías del poblado de Nasahuento ( $17^{\circ} 32^{\prime} \mathrm{S}, 69^{\circ} 35^{\prime} \mathrm{O}, 4.313 \mathrm{~m}$ s.n.m.) y del volcán Guallatire $\left(18^{\circ} 48^{\prime} \mathrm{S}, 69^{\circ} 08^{\prime} \mathrm{O}, 4.750 \mathrm{~m}\right.$ s.n.m.), en la región de Arica y Parinacota, con el objeto de desarrollar cronologías de ancho de anillos en P. tarapacana en Chile. La cronología de Nasahuento se extiendió por 242 años y la de Guallatire por 536 años; ambas fueron comparadas con registros climáticos de su período en común. Los estadígrafos resultantes señalan que las cronologías son adecuadas para fines dendroclimatológicos. El crecimiento estuvo regulado principalmente por las precipitaciones, correlacionándose positivamente con el verano previo y negativamente con el actual, siendo significativo el mes de enero en ambos casos. La temperatura se correlacionó negativamente con el verano previo y positivamente con el actual, evidenciando una relación entre crecimiento y disponibilidad hídrica. Las cronologías desarrolladas en este estudio proveen registros de alta resolución sensibles a la variabilidad climática, lo que permitirá utilizarlas en futuras reconstrucciones climáticas.

Palabras clave: Polylepis tarapacana, dendrocronología, variabilidad climática.

\section{INTRODUCCIÓN}

Los ecosistemas de Los Andes en Sudamérica poseen la particularidad de estar fuertemente influenciados por varios de los forzantes climáticos más importantes del planeta, destacándose dentro de ellos El Niño-Oscilación del Sur (ENSO) y la Oscilación Decenal del Pacífico (PDO). A la vez, los ambientes de alta montaña son una excelente fuente de registros paleoambientales por provenir de espe- cies altamente sensibles a la variabilidad climática, tanto de escala regional como hemisférica (Villalba et al. 1997, Lara et al. 2005a). Los indicadores paleoambientales, tales como los anillos de crecimiento proveen información climática de alta resolución, excediendo con creces los períodos cubiertos por los registros instrumentales realizados por el hombre, que con frecuencia son cortos, fragmentados y poco homogéneos (Luckman 1996, Villalba 2000).

En el contexto de los registros paleoambientales, los 
anillos de los árboles constituyen uno de los registros proxy más importantes por tener una resolución anual e involucrar escalas temporales que son aptas para el estudio de los cambios ambientales presentes y pasados, y estar presentes en todos los continentes con excepción de la Antártida. Esta disciplina científica permite disponer de registros ecológicos y climáticos de alta resolución a partir de las variaciones en el crecimiento del ancho de los anillos de especies leñosas. De esta forma se logra abarcar largos períodos de tiempo, lo que permite proyectarse hacia el pasado en siglos e incluso milenios (Fritts 1976, Bradley y Jones 1992, Lara y Villalba 1993). La dendrocronología ha permitido generar importantes reconstrucciones climáticas con fuerte énfasis en los ecosistemas ubicados en el límite altitudinal arbóreo en ambos hemisferios, demostrando su efectividad como fuente de registros de los cambios climáticos del pasado (Lara et al. 2005b, Boninsegna et al. 2009).

Sin embargo, las regiones subtropicales y semiáridas de América del Sur y gran parte de América tropical, presentan vacíos importantes de registros dendrocronológicos, situación que contrasta con la abundancia y amplia distribución y diversidad de especies leñosas allí presentes (Stahle et al. 2000). En un principio este vacío se atribuyó a la errada creencia de que las especies de clima subtropical no forman anillos de crecimiento anuales, a la existencia de especies poco longevas y a la casi nula preservación de restos leñosos muertos (Bradley y Jones 1992, Boninsegna 1992, Roig 2000). Estudios posteriores determinaron que en las regiones subtropicales de los Andes Centrales (Puna y Prepuna) el crecimiento de las especies leñosas se ve afectado por una fuerte estacionalidad térmica e hídrica, acentuándose a medida que se aumenta en altitud (Roig 2000, Morales et al. 2001). Esta situación provoca un claro reposo vegetativo y la consiguiente formación de anillos anuales bien diferenciados (Villalba et al. 1998). Más aún, en los ambientes de alta montaña el crecimiento es notoriamente más lento, lo que se traduce en una mayor longevidad de las especies allí presentes, y estas mismas condiciones favorecen la preservación de la madera una vez muerta (Roig 2000).

El género Polylepis, familia de las Rosaceae, es endémico de Los Andes centrales, con más de 26 especies distribuidas desde Mérida en Venezuela hasta la provincia de Córdoba en Argentina ( $8^{\circ} \mathrm{N}-32^{\circ} \mathrm{S}$ ) (Kessler 1995ab, Fjeldsa y Kessler 1996, Kessler y Schmidt-Lebuhn 2006). Dentro de las especies del género Polylepis sobresale P. tarapacana que crece ente los $16^{\circ}$ y $23^{\circ} \mathrm{S}$ (Kessler 1995ab). Esta especie forma los bosques o bosquecillos de mayor altitud en el mundo (Braun 1997), encontrándose por sobre $4.200 \mathrm{~m}$ s.n.m y alcanzando elevaciones cercanas a los $5.200 \mathrm{~m}$ en las regiones andinas de Perú, Bolivia, Chile y noreste de Argentina (Kessler 1995ab, Argollo et al 2004).

Las extremas condiciones ambientales donde crece $P$. tarapacana hacen que esta especie posea adaptaciones que le permiten sortear con éxito las bajas temperaturas y la escasez de agua. Una de las adaptaciones más notables es la alta tolerancia al congelamiento, documentándose resistencias en terreno a temperaturas cercanas $-13{ }^{\circ} \mathrm{C}$ para la época seca y fría y de $-6^{\circ} \mathrm{C}$ para la estación húmeda y cálida. Las temperaturas mínimas estimadas para producir daño permanente oscilarían entre -20 y $-21^{\circ} \mathrm{C}$ (Azocar et al. 2007). La severidad del clima también se ve reflejada en las características morfológicas de las plantas, al presentar un follaje siempre verde, compacto, ramas retorcidas y ramificación desde la base y a la vez, cubiertas por una corteza fuertemente laminada a modo de aislante (Kessler 1995ab, Fjeldsa y Kessler 1996). Generalmente se presenta formando rodales puros de baja cobertura que pueden ocupar extensas laderas. Debido a que por siglos fue utilizada como leña y material de construcción, esta especie está considerada en la categoría de "vulnerable" dentro del libro Rojo (Benoit 1989).

La formación de anillos anuales en esta especie motivó la realización de cronologías tanto en Bolivia como en Argentina, demostrando la potencialidad de $P$. tarapacana para ser utilizada en estudios dendroclimatológicos (Argollo et al. 2004, Morales et al. 2004). Debido a lo anterior, Moya (2006) desarrolló las primeras cronologías de ancho de anillos a partir de muestras colectadas en Chile de P. tarapacana, con el objeto de determinar su correlación con las variables climáticas. Por otro lado, la presencia de individuos longevos y la fuerte relación con las variables climáticas, permite llenar el vacío de información dendrocronológica existente en el norte de Chile y que ha sido mencionada como una de las áreas de alta prioridad en América del Sur (Luckman y Boninsegna 2002, Lara et al. 2005a, Boninsegna et al. 2009).

\section{MÉTODOS}

Área de estudio. La región oeste del Altiplano presenta una extrema aridez y gran estabilidad atmosférica (desierto costero) producto de la subsidencia del anticiclón subtropical del Pacífico Sur. En contraste, las tierras bajas al este del Altiplano presentan un régimen continental tropical con un máximo de precipitaciones en los meses de verano (Garreaud y Seluchi 2003). En medio de estas condiciones contrastantes el Altiplano presenta un clima de transición (Garreaud 2000, Garreaud y Seluchi 2003), siendo clasificado por Di Castri y Hajek (1976) como árido de tendencia tropical de altura. Según Fuenzalida (1971), el bioclima imperante en la región alto andina es el tropical xérico, para altitudes sobre $3.000 \mathrm{~m}$ s.n.m. Las precipitaciones en el Altiplano occidental comienzan a ser significativas a medida que se asciende en altitud a causa del mayor aporte de humedad desde el nordeste. Las precipitaciones se concentran en un $80 \%$ durante los meses de verano (noviembre-marzo), con un máximo climatológico en el mes de enero (figura 1). Las precipitaciones se originan a partir de tormentas convectivas que se desarrollan durante la tarde y la noche, siendo episódicas y localizadas (Aceituno y 


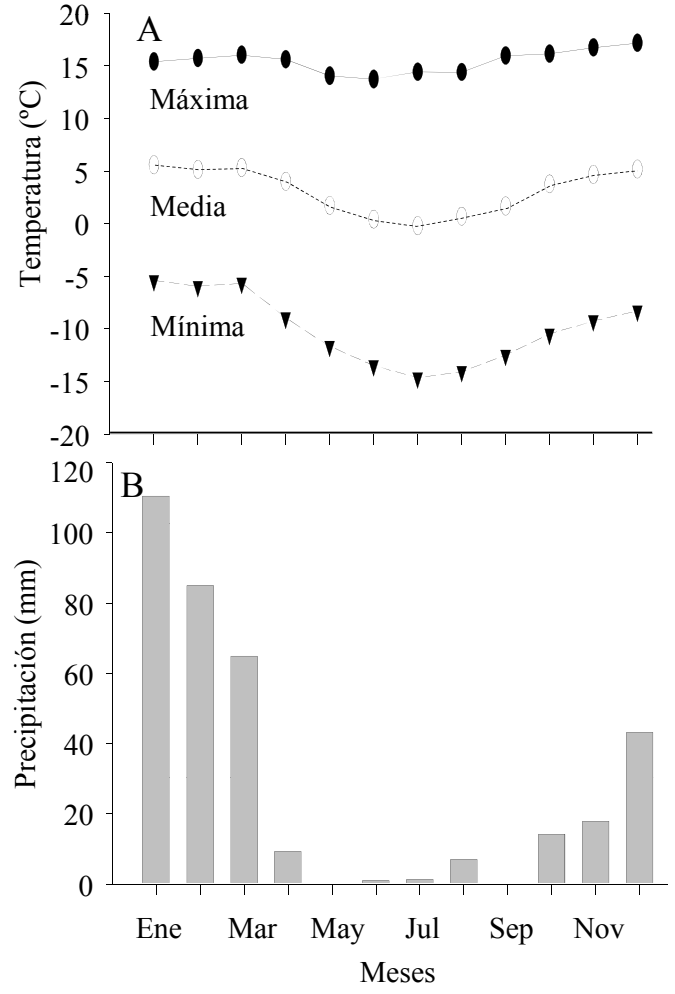

Figura 1. Diagrama climático correspondiente a una media de las estaciones meteorológicas de Caquena, Chungara, Chucuyo, Parinacota, Chilcaya y Guallatire. A) Variaciones mensuales de la temperatura media, media máxima y media mínima y B) distribución de la precipitación mensual a lo largo del año.

Climate diagram for an average of weather stations Caquena, Chungara, Chucuyo, Parinacota Chilcaya and Guallatire. A) Monthly variations in average temperature, average maximum and average minimum, B) distribution of monthly precipitation over the year.

Montecinos 1993, Garreaud 2000, Garreaud et al. 2003, Garreaud y Aceituno 2001). Además, el Altiplano occidental presenta un marcado gradiente de las precipitaciones, disminuyendo de Norte a Sur; mientras en Parinacota $\left(18^{\circ}\right.$ $\left.11^{\prime} \mathrm{S}, 69^{\circ} 33^{\prime} \mathrm{O}\right)$ el promedio anual es de $315 \mathrm{~mm}$, en Ollagüe ( $\left.21^{\circ} 13^{`} \mathrm{~S}, 68^{\circ} 43^{\circ} \mathrm{O}\right)$ alcanza a tan sólo $80 \mathrm{~mm}$.

$\mathrm{La}$ temperatura presenta fluctuaciones diarias muy marcadas debidas principalmente a la alta radiación solar que se produce durante el día y a la pérdida de calor durante la noche, llegando en algunos sitios a más de $30{ }^{\circ} \mathrm{C}$ entre el día y la noche (Aceituno 1993). La temperatura anual promedio es de tan sólo $3,2{ }^{\circ} \mathrm{C}$, siendo las temperaturas medias de verano de $5,2^{\circ} \mathrm{C}$ y en invierno de $1,3{ }^{\circ} \mathrm{C}$ (figura 1). Los suelos están modelados por las condiciones del clima frío y de características xeromórficas del ambiente. Se trata de suelos poco profundos, relativamente jóvenes, compuestos por material particulado como arena y grava gruesa, formados a partir de la descomposición criogénica de la roca madre. En las partes más llanas o en las depresiones intermedias se encuentran suelos formados a partir de materia orgánica en distintas fases de descomposición (bofedales). En cuencas cerradas bajas, los suelos tienen altas concentraciones de sales (salares).

Colecta y preparación de las muestra. Las muestras fueron colectadas en la provincia de Parinacota, de dos rodales próximos a la localidad de Nasahuento $\left(17^{\circ} 32^{\prime} \mathrm{S}, 69^{\circ} 35^{\prime}\right.$ O) y del volcán Guallatire ( $\left.18^{\circ} 48^{\prime} \mathrm{S}, 69^{\circ} 08^{\prime} \mathrm{O}\right)$, a 4.313 y 4.750 m s.n.m., respectivamente. Los sitios están ubicados en la cercanía y dentro del Parque Nacional Lauca, respectivamente. En cada sitio se colectaron con sierra al menos 20 rodelas de las cuales más del 80 \% correspondió a fustes de ejemplares vivos y el resto provino de individuos muertos en pie o restos de material encontrado en el suelo. Sólo se colectaron secciones radiales debido a la dureza de la madera y, principalmente, a lo excéntrico e irregular del crecimiento. Se estimó la altura total de los individuos seleccionados y el número de pies que los constituían. Es importante mencionar que sólo se cortaron fustes de individuos multifustales y no de árboles vivos de un solo pie. Una vez en el laboratorio, las muestras fueron procesadas según los métodos descritos por Stokes y Smiley (1968). Las rodelas fueron emparejadas con un cepillo eléctrico, para luego ser montadas sobre bases de madera y posteriormente pulidas con lijas al agua de diferente granulometría (de 180 a 1.500), hasta lograr distinguir claramente los anillos, los que se diferencian por el aplastamiento radial de las fibras del leño tardío.

Las muestras pulidas se analizaron bajo lupa estereoscópica para realizar el conteo de los anillos. Luego se midió el ancho de los anillos de crecimiento utilizando un dendrómetro Velmex conectado a un computador con una precisión de 0,001 mm (Robinson y Evans 1980). Para las rodelas de árboles vivos, se adoptó la convención de Schulman (1956), para el hemisferio Sur, que asigna a cada anillo de crecimiento la fecha en que se dio inicio al crecimiento (primavera). De esta forma, el anillo que comenzó a crecer en la primavera del 2002, se fechó como correspondiente a ese año.

Una vez generados los archivos digitales con las mediciones de los anillos se ingresaron al programa COFECHA, que permite comprobar y corregir el cofechado permitiendo detectar la presencia de anillos falsos o ausentes (Holmes 1983). Las series de anillos correctamente fechadas fueron estandarizadas con la utilización del programa ARSTAN (Cook 1985), el cual permite remover la tendencia en el crecimiento no atribuible a la variación climática (Fritts 1976). Las series estandarizadas fueron promediadas obteniéndose una cronología media para cada uno de los dos sitios estudiados, constituyendo cada cronología una serie temporal que representa las variaciones anuales en el crecimiento radial de los árboles de P. tarapacana en los dos sitios muestreados. De acuerdo a los objetivos 
de este estudio se utilizó un método de estandarización en que las series de anchos de anillos se ajustaron a una curva exponencial negativa, a una regresión lineal negativa o una línea horizontal, de acuerdo al mejor ajuste para cada una de las series. Las cronologías fueron desarrolladas utilizando las versiones estándar y residual.

La versión estándar se caracteriza por preservar la señal de baja frecuencia, mientras que la cronología residual conserva la señal de alta frecuencia lo que la hace recomendable en los estudios dendroclimatológicos, En este último caso se remueve la auto correlación presente en las series de ancho de anillos (componente biológico), con la finalidad de determinar el correcto nivel de significancia de algunos estadísticos.

Análisis de las relaciones entre el clima y el crecimiento anual. Las cronologías residuales de ambos sitios fueron comparadas con los registros metereológicos (precipitación y temperatura) para determinar que parámetros ambientales están influenciando el crecimiento de P. tarapacana. Este método consiste en correlacionar las variaciones interanuales en el ancho de los anillos de crecimiento con las fluctuaciones climáticas interanuales mes a mes. Esta relación es examinada en un período común entre la cronología y los registros instrumentales. A esto se le conoce como función de correlación (Blasing et al. 1984) y se calculó utilizando el programa RESPO versión 6.00 (Holmes 1983). Debido a que el crecimiento de los anillos puede estar influenciado por las condiciones climáticas previas a la formación de los anillos (Fritts 1976), en el análisis se incluyó el período de tiempo que abarca tanto la estación de crecimiento actual como la previa. Consecuentemente, la correlación entre el ancho de los anillos y los datos climáticos se calculó para 17 meses, comenzando en enero de la estación de crecimiento previa y finalizando en mayo de la estación de crecimiento actual. Con el fin de evaluar la variabilidad temporal y la fortaleza de la señal común de las cronologías, se utilizó la rutina Rbar (Running series of average correlations) y EPS (Expressed population signal) (Briffa 1995). Siendo Rbar el índice de correlación promedio de todos los posibles pares de muestras con una ventana de tiempo determinada, y el EPS, la señal poblacional entre una cronología dada y una cronología hipotética que ha sido infinitamente replicada para un intervalo de tiempo común (Wigley et al. 1984).
Las estaciones meteorológicas utilizadas fueron las de Oruro ( $17^{\circ} 57^{\prime} \mathrm{S}, 67^{\circ} 08^{\prime} \mathrm{O}$ a $3.706 \mathrm{~m}$ s.n.m), Uyuni $\left(20^{\circ} 28^{\prime}\right.$ S, $66^{\circ} 48^{\prime} \mathrm{O}, 3.660 \mathrm{~m}$ s.n.m), Colcha $\left(20^{\circ} 47^{\prime} \mathrm{S}, 67^{\circ} 47^{\prime} \mathrm{O}\right.$, 3.700 m s.n.m) y Calcha de Lipez $\left(21^{\circ} 01^{\prime} \mathrm{S}, 67^{\circ} 58^{\prime} \mathrm{O}\right.$, 3.670 m s.n.m) de Bolivia para el período 1944 - 2000. Las estaciones meteorológicas bolivianas fueron seleccionadas debido a que poseen los registros climáticos más extensos y homogéneos.

\section{RESULTADOS}

Características de las cronologías. Con las muestras colectadas en el sitio Nasahuento (NAS), se generó una cronología de 242 años a partir de 14 series co-fechadas, correspondiendo al $58 \%$ del total de las muestras en ese sitio. Por otra parte, la cronología de Guallatire (GUA), tiene una extensión de 536 años y está conformada por 17 series, equivalentes al $68 \%$ del total de los árboles colectados (cuadro 1).

Las muestras colectadas en Nasahuento presentaron un promedio de edad de 107 años, con un rango entre 53 y 216 años, encontrándose sólo tres segmentos con problemas potenciales de fechado. En el caso de Guallatire se obtuvo un largo promedio de radios de 236 años, con un rango entre 56 y 422 años, con 14 segmentos con posibles problemas de fechado.

Los estadígrafos que caracterizan las cronologías mostraron una fuerte similitud entre ambas, destacando una mayor señal común en el sitio Nasahuento por sobre Guallatire en todas las variables analizadas (cuadro 2).

La cronología Nasahuento en su versión estándar presenta tres períodos de crecimiento por debajo de la media, siendo dos de ellos de corta duración (1761 a 1780 y 1825 a 1835) y un tercero comprendido entre los años 1860 y 1940, más notorio y pronunciado, para luego en el período inmediatamente después, presentar un crecimiento por sobre la media entre los años 1950 y 1980. Finalmente destaca una disminución del ancho de los anillos a partir de la década de los 80's (figura 2).

La cronología del sitio Guallatire muestra tres períodos de crecimiento por debajo de la media, de una mayor duración que los presentados en el sitio Nasahuento, correspondiendo a los años 1540 a 1600, 1680 a 1780 y desde la década de los 80 ' hasta el último año de formación de los anillos (2002). Igualmente, destacan tres períodos de cre-

Cuadro 1. Amplitud y número de árboles incluidos en las cronologías. Extent and number of trees included in the chronologies.

\begin{tabular}{|c|c|c|c|c|c|c|c|c|c|}
\hline \multirow{2}{*}{ Sitio } & \multirow{2}{*}{ Código } & \multirow{2}{*}{ Ubicación } & \multicolumn{2}{|c|}{$\mathrm{N}^{\mathrm{o}}$ árboles } & \multirow{2}{*}{$\begin{array}{c}\mathrm{N}^{\circ} \text { total series } \\
\text { medidas }\end{array}$} & \multirow{2}{*}{$\begin{array}{c}\mathrm{N}^{\circ} \text { series } \\
\text { cofechadas }\end{array}$} & \multicolumn{2}{|c|}{ Amplitud (años) } & \multirow{2}{*}{ Años } \\
\hline & & & Vivos & Muertos & & & Inicio & Final & \\
\hline Nasahuento & NAS & $\begin{array}{l}17^{\circ} 32^{`} \mathrm{~S} \\
69^{\circ} 35^{`} \mathrm{O}\end{array}$ & 23 & 1 & 25 & 14 & 1761 & 2002 & 242 \\
\hline Guallatire & GUA & $\begin{array}{l}18^{\circ} 48^{`} \mathrm{~S} \\
69^{\circ} 08^{`} \mathrm{O}\end{array}$ & 20 & 5 & 28 & 17 & 1467 & 2002 & 536 \\
\hline
\end{tabular}


Cuadro 2. Resumen de los estadígrafos para las cronologías Nasahuento y Guallatire. Los estadígrafos son para las cronologías estándares.

\begin{tabular}{ccccccc}
\multicolumn{7}{c}{ Descriptive statistics for the two standard chronologies, Nasahuento and Guallatire. } \\
\hline Sitio & Código & $\begin{array}{c}\text { Sensibilidad } \\
\text { media }\end{array}$ & $\begin{array}{c}\text { Correlación } \\
\text { entre series* }\end{array}$ & $\begin{array}{c}\text { Auto correlación } \\
\text { de } 1^{\text {er }} \text { orden }\end{array}$ & $\begin{array}{c}\text { Varianza del 1 } 1^{\text {er }} \text { auto } \\
\text { vector (\%) }\end{array}$ & $\begin{array}{c}\text { Correlación media } \\
\text { entre árboles* }\end{array}$ \\
\hline $\begin{array}{c}\text { Nasahuento } \\
\text { Guallatire }\end{array}$ & NAS & 0,211 & 0,378 & 0,593 & 46,99 & 0,364 \\
\hline
\end{tabular}

$* P<0,005$.

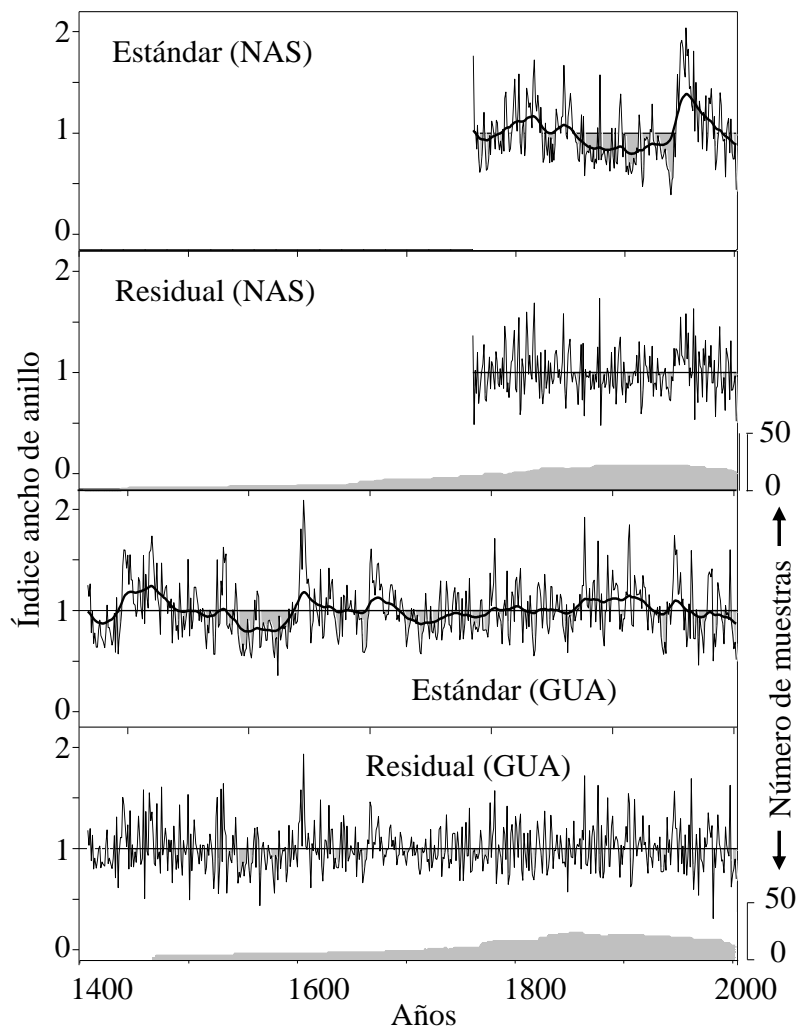

Figura 2. Variación de índices de anillos de crecimiento para Nasahuento y Guallatire en la versión estándar y residual con número de muestras incluidas en la cronología. La línea gruesa en las cronologías estándares resulta de un filtrado exponencial de los índices originales (Rosenbluth et al. 1997).

Variation in rates of tree rings growth in Nasahuento and Guallatire residual standard and number of samples included in the chronology. The line is an exponential filter (Rosenbluth et al. 1997).

cimiento por sobre la media, correspondiendo a los años 1450 a 1490,1610 a 1680 y 1880 a 1940 . Ambas cronologías presentaron una alta variabilidad interanual (figura 2).

De la comparación de ambas cronologías en su versión estándar, se destacan las coincidencias de los eventos extremos, tanto sobre como bajo la media (por ejemplo, bajo y alto crecimiento en 1941, y 1953, respectivamente). La matriz de correlación de Nasahuento y Guallatire mostró que ambos sitios están correlacionados significativamente $(r=0,45 ; P<0,005)$. No obstante, las cronologías estándar las cuales preservan la señal de baja frecuencia, muestran

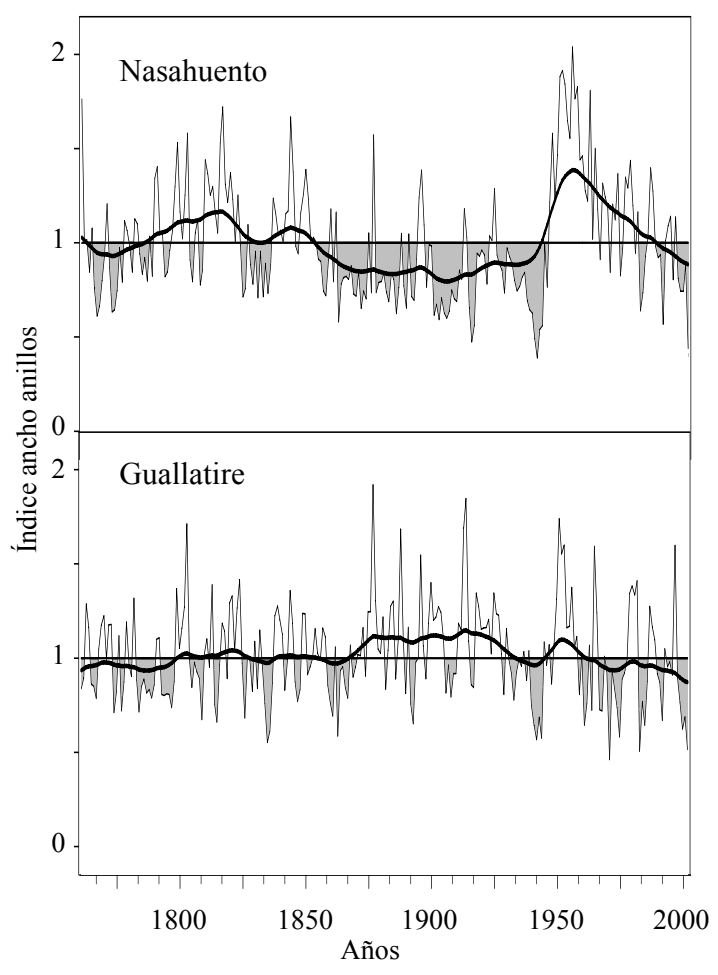

Figura 3. Variación del índice de ancho de anillos para las cronologías Nasahuento y Guallatire en su versión estándar para el período común entre 1761 y 2002.

Variation of the index of tree rings width for the chronologies Nasahuento and Guallatire in their standard version for the common period between 1761 and 2002.

tendencias opuestas en ambos sitios para el período 18801930. Además, el abrupto incremento en el crecimiento de Nasahuento en el período 1950-1975, ausente en Guallatire, es un reflejo de que este incremento sea un factor local no climático que afectó a este sitio, por ejemplo corta de árboles (figura 3).

En relación al estadístico EPS, la cronología Nasahuento presentó valores mayores a 0,85 a partir de 1875 y la cronología Guallatire a partir de 1560 (Wigley et al. 1984, Briffa 1995). Esto indica que hay una señal común adecuada, salvo en el período anterior a 1860 en la cronología Nasahuento y desde 1460 a 1550 , y 1730 a 1770 en Guallatire debido al bajo número de muestras incluidas 
en esos períodos (menor a seis muestras, figura 4).

Correlación con las variables climáticas. Se observó buena respuesta en el crecimiento de $P$. tarapacana frente a las variables climáticas de temperatura y precipitación, analizada mediante las correlaciones entre las cronologías Nasahuento y Guallatire y los registros instrumentales. Ambas cronologías presentaron correlaciones altas con la temperatura del verano actual, estando el crecimiento de $P$. tarapacana positivamente correlacionado con los meses de diciembre, enero y febrero (figura 5). Por otra parte, en el verano previo el crecimiento estuvo correlacionado en forma negativa con las temperaturas (enero en Nasahuento y enero, marzo, abril y mayo en Guallatire), presentando las relaciones más fuertes y significativas en los meses de enero previo y actual.

El sitio Nasahuento mostró una correlación positiva con la precipitación durante el mes de enero previo y
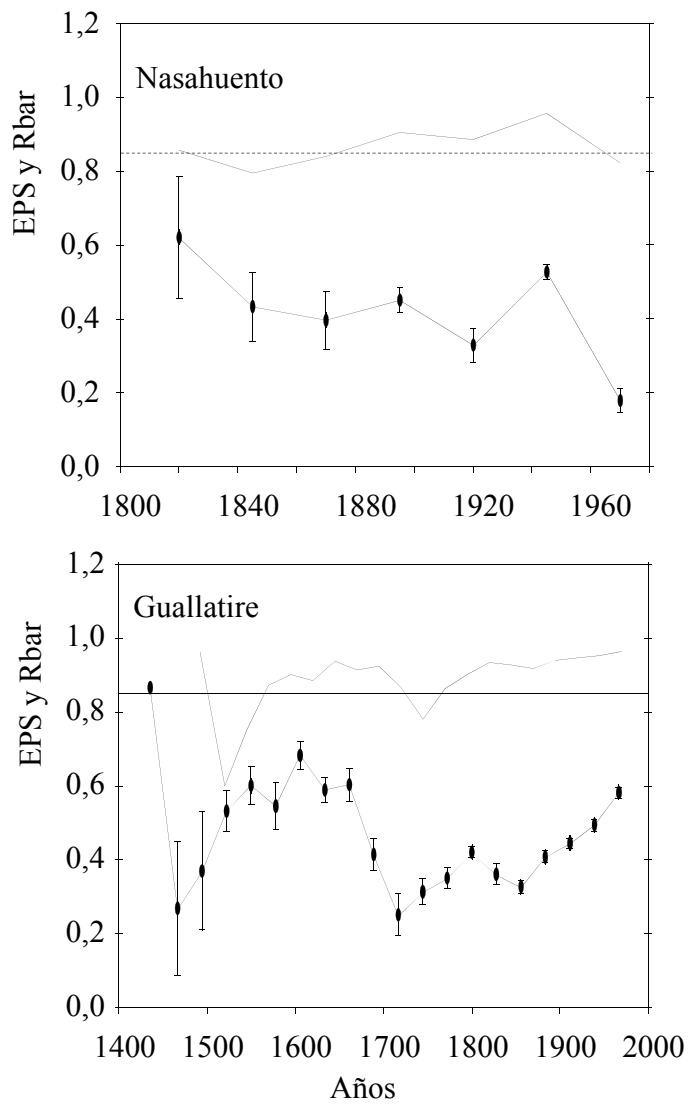

Figura 4. Estadígrafos Rbar con ventana móvil de 50 años traslapados en 25 años y EPS para las cronologías de Nasahuento y Guallatire.

Moving mean correlation coefficient (Rbar) and Expressed Population Signal (EPS) statistics for the composite chronologies using a 50-year window with a 25-year overlap for the chronology Nasahuento and Guallatire. negativa con igual mes de la estación actual, estando ambas correlacionadas significativamente. Por otra parte, el crecimiento de P. tarapacana del sitio Guallatire mostró correlaciones positivas con los meses de enero previo y abril actual, presentando correlación negativa con el crecimiento en los meses de enero y febrero actual (figura 5).

\section{DISCUSIÓN Y CONCLUSIONES}

Las variaciones interanuales en las rigurosas condiciones ambientales donde crece $P$. tarapacana se ven reflejadas en el crecimiento de los anillos de sus individuos, haciendo de $P$. tarapacana una fuente importante de registros dendrocronológicos para el Altiplano (Argollo et al. 2004, Christie et al. 2009). Igualmente los períodos de supresión y liberación en ambas cronologías destacan las coincidencias de los eventos extremos, tanto por sobre como por bajo la media, permitiendo establecer a la vari-

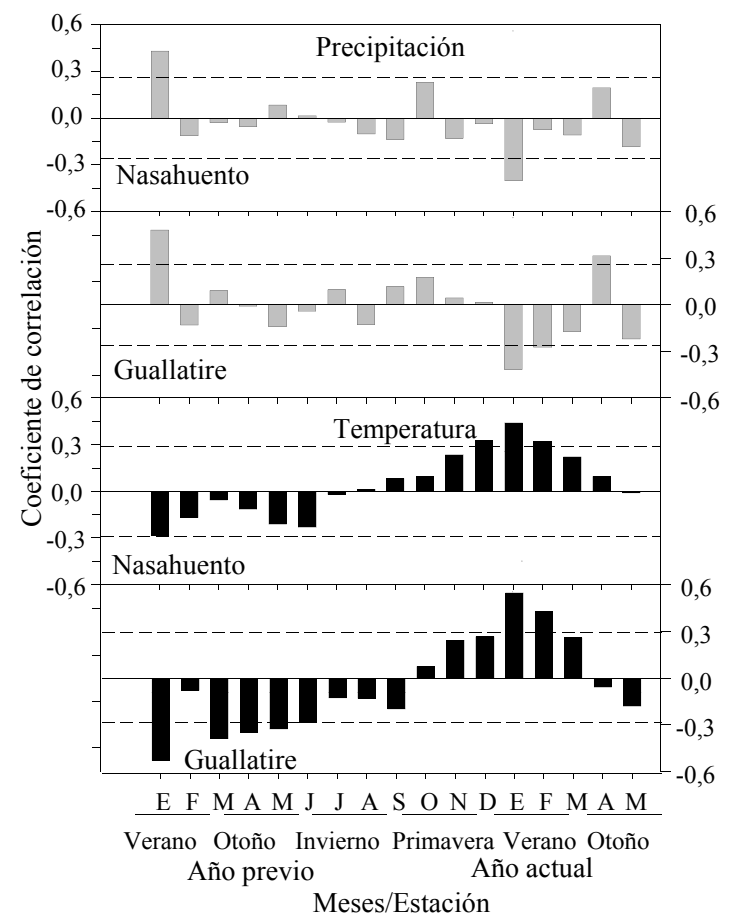

Figura 5. Funciones de correlación entre la temperatura media de Oruro (Bolivia), la precipitación promedio de las estaciones meteorológicas de Oruro, Uyuni, Calcha y Colcha de Lipez (Bolivia), y las cronologías de ancho de anillos de crecimiento de $P$. tarapacana para la localidad de Nasahuento y Guallatire, para el período 1944-2000. Las líneas segmentadas corresponden a los límites de confianza al $95 \%$.

Correlation function between the average temperature of Oruro (Bolivia), the average rainfall of meteorological stations of Oruro, Uyuni, Calcha and Colcha de Lipez (Bolivia), and tree ring- width chronologies of P. tarapacana for the locality of Nasahuento (NAS) and Guallatire (GUA), for the period 1944-2000. The dotted lines correspond to confidence limits at $95 \%$. 
abilidad climática como ente regulador del crecimiento de P. tarapacana.

Este trabajo presenta las primeras cronologías de ancho de anillos en P. tarapacana, a partir de colecciones realizadas en el año 2003 en dos localidades ubicadas a más de $4.200 \mathrm{~m}$ de altitud al interior de la provincia de Parinacota, Chile. Las cronologías de este trabajo no sólo se destacan por ser las primeras en la región, sino también por ser las de mayor altitud y las más septentrionales de Chile. Los estadígrafos resultantes de la medición de la calidad de las cronologías (cuadro 2) indican que ambas cronologías son adecuadas para ser utilizadas en el estudio de la variabilidad climática de la región. La sensibilidad media en ambos sitios es similar a las reportadas para cronologías en Bolivia y Argentina para esta misma especie (Argollo et al. 2004). A la vez, estos valores son similares a los descritos para otras especies subtropicales de Sudamérica (Villalba et al. 1987, 1992) y superiores al de las especies de zonas templadas y frías (Boninsegna 1992), reflejando la calidad de ambas cronologías.

La autocorrelación de primer orden en ambos sitios resultó alta (cuadro 2), reflejando la fuerte influencia biológica que tienen las condiciones climáticas del año previo sobre el crecimiento radial de esta especie. En las cronologías desarrolladas en el Altiplano boliviano esta variable igualmente resultó alta con valores similares, destacando el sitio Sajama con una autocorrelación de 0,48 (Argollo et al. 2004), el más cercano a los sitios de este estudio.

La correlación entre árboles igualmente resultó alta, coincidiendo en ambos sitios con un coeficiente medio de correlación de $r=0,36$, lo cual demuestra la existencia de una importante señal común que estaría rigiendo el crecimiento sincrónico de los árboles. Esta variable, una de las más útiles al momento de evaluar la calidad de las cronologías, confirma la presencia de una señal común con condiciones homogéneas que se verían reflejadas en el crecimiento de un gran número de árboles en los sitios de estudio. Resultados similares fueron encontrados por Argollo et al. (2004), Soliz et al. (2009) y Christie et al. (2009), confirmando una clara señal común que influencia el crecimiento de P. tarapacana, tanto para los individuos dentro de los rodales como en todo el Altiplano. El análisis anterior indica que los estadísticos obtenidos en las cronologías de Nasahuento y Guallatire son adecuados y comparables a los de estudios anteriores incluyendo 14 y 17 series, respectivamente. Futuras investigaciones debieran evaluar si es que al aumentar el número de series es posible mejorar los estadísticos de las cronologías de P. tarapacana, respecto a lo logrado hasta ahora. El significativo coeficiente de correlación entre Nasahuento y Guallatire permite constatar el grado de similitud en el patrón de crecimiento de ambas cronologías y confirmar la existencia de una marcada señal común que está influyendo en el crecimiento de P. tarapacana en los dos sitios estudiados. La alta correlación también se ve reflejada grá- ficamente en la coincidencia de anillos delgados y gruesos. En ellas, los eventos extremos coinciden con anillos marcadores los cuales son comunes en las cronologías Nasahuento y Guallatire.

Las funciones de correlación indican que el crecimiento de $P$. tarapacana está significativamente influenciado por las precipitaciones del mes de enero tanto en el verano previo como en el actual, pero en sentido inverso. La precipitación de diciembre y enero del verano previo están positivamente correlacionada con el crecimiento. Por el contrario, el crecimiento se ve negativamente correlacionado con las precipitaciones del mes de enero del verano actual.

El crecimiento presentó correlaciones negativas con las temperaturas en el verano previo y positivas durante con el verano actual (diciembre a febrero). De esta forma, el crecimiento de P. tarapacana se vio favorecido con temperaturas por sobre la media en la estación de crecimiento actual, y por temperaturas por debajo de la media en la estación previa, coincidiendo con los resultados obtenidos en Bolivia, Argentina y Chile (Argollo et al. 2004, Morales et al. 2004, Christie et al. 2009).

Dado el carácter árido del Altiplano, es posible encontrar una mayor influencia de la precipitación que de la temperatura sobre el crecimiento, lo que no se ve directamente en las funciones de correlación (figura 5) La aparente mayor influencia de las temperaturas que de las precipitaciones puede obedecer a la naturaleza de los campos climáticos de temperatura y precipitación en el Altiplano.

Los campos de temperatura en el Altiplano son mucho más extensos y homogéneos que los de la precipitación (Garreaud y Seluchi 2003). A consecuencia de esta homogeneidad, los sitios de muestreo pueden estar distantes del punto de toma de datos y, sin embargo, compartir el mismo campo de variaciones de temperatura. Por el contrario, esta situación no es válida para la precipitación cuyo campo es mucho más variable espacialmente. Las precitaciones del Altiplano, son de carácter convectivas, episódicas y espacialmente localizadas y, por lo tanto, altamente variables. Debido a esto, sólo los sitios de colecta muy cercanos a las estaciones meteorológicas pueden considerarse confiables en su relación con la precipitación (Vuille 1999, Garreaud 2000).

Los estudios realizados por Argollo et al. (2004), Soliz et al. (2009) y Christie et al. (2009), tanto en Bolivia como en Argentina, señalan que en el Altiplano el crecimiento de $P$. tarapacana está controlado principalmente por las precipitaciones, durante los meses del verano previo y particularmente en el mes de enero. En cambio, para ese mismo período las altas temperaturas interfieren negativamente con el crecimiento. La relación inversa entre las precipitaciones y las temperaturas se debe al efecto negativo de las altas temperaturas en la disponibilidad hídrica para las plantas, lo que se ve reflejado en un menor crecimiento. De esta forma las precipitaciones del verano previo constituyen un reservorio para el crecimiento en la estación siguiente. 
Las respuestas opuestas en el verano previo y actual para la precipitación y la temperatura es difícil de explicar. Para dar respuesta a esta interrogante, es necesario desarrollar estudios eco-fisiológicos de largo plazo con P. tarapacana, que nos permitan entender plenamente la biología de especies creciendo en ambientes climáticos extremos.

Las cronologías desarrolladas demuestran la potencialidad de esta especie para ser utilizada con fines dendroclimáticos, ya que además de formar anillos anuales bien diferenciados y estar influenciadas por las variaciones climáticas a escala regional presenta individuos longevos de más de 600 años (árbol vivo encontrado en la localidad de Collacahua, al sur de Guallatire, no incluido en este estudio). Esta situación más la posibilidad de extraer material de antiguas construcciones (poblados abandonados, antiguas iglesias y material enterrado), demuestra las excelentes perspectivas para el desarrollo de futuras cronologías y posteriores reconstrucciones climáticas (Argollo et al. 2004, Soliz et al. 2009, Christie et al 2009).

Polylepis tarapacana abre grandes posibilidades para continuar avanzando en la reconstrucción de las variables climáticas pasadas, permitiendo que amplias regiones subtropicales carentes de registros paleoambientales puedan ser incluidas en los estudios dendroclimáticos. Esta especie permite avanzar con registros dendrocronológicos en más de siete grados de latitud hacia el Ecuador desde los $23^{\circ}$ hasta los $16^{\circ} \mathrm{S}$, obteniéndose valiosa información de la región alto andina del sur del Perú, parte de Bolivia, norte de Chile y noreste de Argentina. Además, el género Polylepis, debido a su amplia distribución y al estar ubicados a más de 1.700 m s.n.m., permitirá seguir incluyendo nuevas regiones y de esta forma completar el vacío de registros ambientales en la transecta Polo-Ecuador-Polo a través de las Américas. El desarrollo de otros estudios como éste permitirá la realización de futuras reconstrucciones climáticas las que, a la vez, permitirán comprender si los cambios ocurridos en la actualidad son cíclicos y naturales o son producto de la actividad humana.

\section{REFERENCIAS}

Aceituno P. 1993. Elementos del clima en el Altiplano Sudamericano. Revista de Geofísica 44, 37-55.

Aceituno P, A Montecinos. 1993. Circulation anomalies associated with dry and wet periods in the South American Altiplano. Preprints $4^{\text {th }}$ Int. Conf. on Southern Hemisphere Meteorology and Oceanography, Hobart, Australia. American Meteorogical Society. p. 330-331.

Argollo J, C Soliz, R Villalba. 2004. Potencialidad dendrocronológica de Polylepis tarapacana en los Andes Centrales de Bolivia. Ecología en Bolivia 39: 5-24.

Azocar A, F Rada, C García-Núñez. 2007. Functional characteristics of the arborescent genus Polylepis along a latitudinal gradient in the high Andes. Interciencia 32(10): 663-668.

Benoit C. 1989. Red book of Chilean terrestrial flora. Santiago, Chile. Corporación Nacional Forestal (CONAF). 151 p.

Blasing TJ, AM Solomon, DN Duvick. 1984. Response functions revisited. Tree-Ring Bulletin 44: 1-15.

Boninsegna JA, J Argollo, JC Aravena. J Barichivich, D Christie, ME Ferrero, A Lara, C Le Quesne, BH Luckman, M Masiokas, M Morales, JM Oliveira, F Roig, A Srur, R Villalba. 2009. Dendroclimatological Reconstructions in South America: A review. Paleogeography, Paleoclimatology, Palaecology 281: 210-228.

Boninsegna JA. 1992. South American dendroclimatological records, In Bradley RS, PD Jones eds. Climate since A. D. 1500. Routledge, London, UK. p. 446-462.

Bradley RS, PD Jones. 1992. Climate Since A. D: 1500: Introduction. In Bradley RS, PD Jones eds. Climate since A. D. 1500. Routledge, London, UK. p.1-16.

Braun G. 1997. The use of digital methods in assessing forest patterns in an Andean environment: the Polylepis example. Mountain Research and Development 17: 253-262.

Briffa KR. 1995. Interpreting high-resolution proxy climate data: the example of dendroclimatology. In Von Storch H, A Navarra eds. Analysis of climate variability, applications of statistical techniques. Berlin, Alemania. Springer. p. 77-94.

Christie D, A Lara, J Barichivich, R Villalba, MS Morales, E Cuq. 2009. El Niño-Southern Oscilation signal in the world`s high-elevation tree-ring chronologies from the Altiplano, Central Andes. Paleogeography, Paleoclimatology, Palaecology 281, 309-319.

Cook ER. 1985. A time series analysis: Approach to tree-ring standardization. Ph. D. Dissertation. Tucson, USA, University of Arizona. $171 \mathrm{p}$.

Di Castri F, E Hajek. 1976. Bioclimatología de Chile. Santiago, Chile. Vicerrectoria Académica de la Universidad Católica de Chile. 124 p.

Fjedsa J, M Kessler. 1996. Conserving the biological diversity of Polylepis Woodlands of the Highland of Peru and Bolivia. A contribution to sustainable natural resource management in the Andes.. Copenhagen, Denmark. NORDECO. 250 p.

Fritts HC. 1976. Tree-rings and climate. Arizona, USA. Laboratory of Tree-Ring Research. University of Arizona. 567 p.

Fuenzalida H. 1971. Climatología de Chile. Publicación interna de la sección de Metereología. Departamento de Geofísica y Geodesia, Universidad de Chile. Santiago, Chile. 73 p.

Garreaud R, M Seluchi. 2003. Pronóstico de la convección en el Altiplano Sud Américano empleando el modelo regional ETA/CPTEC. Meteorológica 26: 25-38.

Garreaud R, M Vuille, C Clement. 2003. The climate of the Altiplano: Observed current conditions and past changes mechanisms. Paleogeography, Paleoclimatology, Palaecology 3054: 1-18.

Garreaud R, P Aceituno. 2001. Interannual rainfall variability over the South American Altiplano. Journal of Climate 14: 2779-2789.

Garreaud R. 2000. Intraseasonal Variability of moisture and rainfall over the South American Altiplano. Monthly Weather Review 128: 3337-3346.

Holmes RL. 1983. Computer assisted quality control in tree-ring dating and measurements. Tree-ring Bulletin 43: 69-75.

Kessler M, AN Schmidt-Lebuhn 2006. Taxonomical and distributional notes on Polylepis (Rosaceae). Organisms Diversity and Evolution 1: 1-10.

Kessler M. 1995a. Revalidación de Polylepis regulosa Bitter (Rosaceae). Gayana Botanica 52(2): 49-51.

Kessler M. 1995ㅁ. The genus Polylepis (Rosaceae) in Bolivia. Conservatoire et Jardin Botaniques de Geneve. Candollea 50. 172 p. 
Lara A, A Wolodarsky-Franke, JC Aravena, R Villalba, ME Solari, L Pezoa, A Rivera, C Le Quesne. 2005a. Climate fluctuations derived from tree-ring other proxy-records in the Chilean Andes: state of the art and future prospects $\mathrm{In} \mathrm{Hu}$ ber UM, HK Bugmann, MA Reasoner eds. Global change and mountain regions: An overview of current knowledge. Dordrecht, The Netherlands. Springer. p. 145-156.

Lara A, R Villalba, A Wolodarsky-Franke, JC. Aravena, BH Luckman, E Cuq. 2005b. Spatial and temporal variation in Nothofagus pumilio Growth at treeline along its latitudinal range $\left(35^{\circ} 40^{\prime}-55^{\circ} \mathrm{S}\right)$ in the Chilean Andes. Journal of Biogeography 31: 1-17.

Lara A, R Villalba. 1993. “A 3620 year temperature reconstruction from Fitzroya cupressoides tree rings in Southern South America”. Science 260: 1104-1106.

Luckman BH, JA Boninsegna. 2002. Evaluación de las variables climáticas presente, pasada y futura en las Américas a partir del medio ambiente del límite del bosque. IAI Newsletter 27: 5-9.

Luckman BH, 1996. Dendroglaciology at Peyto Glacier, Alberta, Canada. In Dean JS, DM Meko, TW Swetnam eds. Tree Rings, Environment and Humanity. Radiocarbon. p. 679688.

Morales MS, R Villalba, HR Grau, L Paolini. 2004. Rainfall controlled tree growth in high elevation subtropical treelines. Ecology 85(11): 3080 - 3089.

Morales MS, R Villalba, HR Grau, P Villagra, JA Boninsegna, A Ripalta, L Paolini. 2001. Potencialidad de Prosopis ferox Griseb (Leguminosae, subfamilia: Mimosoideae) para estudios dendrocronológicos en los desiertos subtropicales de alta montaña. Revista Chilena de Historia Natural 74: 865-872.

Moya J. 2006. Desarrollo de las primeras cronologías de Queñoa (Polylepis tarapacana Phil.) en el Altiplano de la región de Tarapacá, Chile. Tesis Magíster en Ciencias. Valdivia, Chile. Facultad de Ciencias Forestales, Universidad Austral de Chile. 73 p.

Roig F. 2000. Dendrocronología en los bosques del Neotrópico: Revisión y prospección futura. In Roig F eds. Dendrocronología en América Latina. Mendoza, Argentina. EDIUNC. p. 307-355.

Robinson WJ, R Evans. 1980. A microcomputer-based tree-ring measuring system. Tree-ring Bulletin 40: 59-63.
Rosenbluth BH, A Fuenzalida, P Aceituno. 1997. Recent temperature variations in Southern South America. International Journal of Climatology 17: 67-85.

Schulman E. 1956. Dendroclimatic changes in semiarid America. University of Arizona Press, Tucson Arizona, USA. 142 p.

Soliz C, R Villalba, J Argollo, MS Morales, D Christie, J Moya, J Pacaje. 2009. Spatio-temporal variations in Polylepis tarapacana radial growth across the Bolivian Altiplano during the 20th century. Paleogeography, Paleoclimatology, $\mathrm{Pa}$ laecology 281: 296-308.

Stahle D, J Villanueva, MK Cleaveland, MD Therrell, GJ Paull, BT Burns, W Salinas, H Suzan, PZ Fule. 2000. Recent treering research in México. In Roig F eds. Dendrocronología en América Latina. Mendoza, Argentina. EDIUNC p. 285-306.

Stokes MA, TL Smiley. 1968. An introduction to tree-ring dating. The University of Chicago Press, Chicago, Illinois, USA. p. 73.

Villalba R. 2000. Dendroclimatology: a Southern Hemisphere Perspective. In Smolka PP, W Volkheimer eds. Paleo and Neoclimates of the Southern hemisphere: the state of the arts. Berlin, Germany. Springer. p. 28-57.

Villalba R, HR Grau, JA Boninsegna, GC Jacoby, A Ripalta. 1998. Climatic variations in subtropical South America inferred from upper-elevation tree-ring records. International Journal of Climatology 18: 1463-1478.

Villalba R, JA Boninsegna, TT Veblen, A Schmelter, S Rubulis. 1997. Recent trends in tree-ring records from high elevation sites in the Andes of northern Patagonia. Climatic Change 36: 425-454.

Villalba R, RL Holmes, JA Boninsegna. 1992. Spatial patterns of climate and tree growth anomalies in subtropical Northwestern Argentina. Journal of Biogeography 19: 631-649.

Villalba R, JA Boninsegna, A Ripalta. 1987. Climate site conditions and tree-growth subtropical northwestern Argentina. Canadian Journal Forest Research 17:1527-1544.

Vuille M. 1999. Atmospheric circulation over the Bolivian Altiplano during dry and wet periods and extreme phases of the Southern Oscillation. International Journal of Climatology 19: 1579-1600.

Wigley TML, KR Briffa, PD. Jones. 1984. On the average value of correlated time series, with applications in dendroclimatology and hydrometeorology. Journal of Climate and Applied Meteorology 23: 201-213. 
